\title{
ANÁLISIS DE LOS MOVIMIENTOS DE LAS OPERACIONES DE LAS GRÚAS RTG EN PUERTOS DE PANAMÁ USANDO SIMULADOR E-TECH
}

\author{
Jahiro Sutherland ${ }^{1}$; \\ Yoan Martínez-López²; \\ Carlos A. Rovetto ${ }^{3}$; \\ Elia E. Cano ${ }^{4}$
}

\begin{abstract}
The containers use trend and cranes integration for moving them, have a huge impact in the physic design of existing ports in the world. In this document a comparison research between rectilinear movement (inverted $U$ ) and parabolic movement (overlapping) is presented by using a rubber tyred gantry (RTG) crane simulator. For the tests analysis, the chi-quarter method was employed, showing up that operators presents less difficult handling parabolic movement than rectilinear one.
\end{abstract}

Keywords: RTG crane; gantry; hoist, simulation; operators.

Resumen: La aparición del uso del contenedor y la incorporación de grúas para el movimiento de estos, causó una auténtica revolución en el diseño de los puertos existentes cambiando su aspecto físico. Este trabajo establece un estudio de comparación entre el movimiento de $\mathrm{U}$ invertida (rectilínea) y el movimiento de Overlapping (parabólica) a través del uso de una herramienta de simulación de grúas pórtico de patio sobre neumáticos de goma (RTG). Para la

\footnotetext{
${ }^{1}$ Facultad de Ciencias y Tecnología - Universidad Tecnológica de Panamá (UTP) Panamá - Panamá. jazael.01@ hotmail.com

${ }^{2}$ Facultad de Informática y Ciencias Exactas - Universidad de Camagüey (UC) Camagüey - Cuba. yoan.martinez@ reduc.edu.cu

${ }_{3}^{3}$ Facultad de Ingeniería de Sistemas Computacionales - Universidad Tecnológica de Panamá (UTP) Panamá Panamá. carlos.rovetto@utp.ac.pa

${ }^{4}$ Facultad de Ingeniería de Sistemas Computacionales - Universidad Tecnológica de Panamá (UTP) Panamá Panamá. elia.cano@utp.ac.pa
}

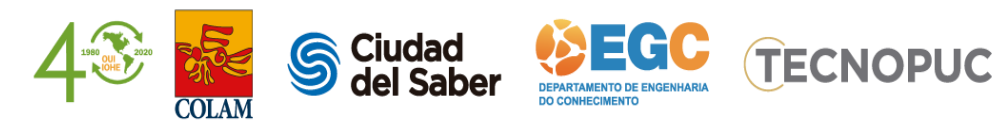




\section{ciki}

X Congreso internacional de conocimiento e innovación
Ciudad del Saber, Panamá

19 y 20 de noviembre 2020

evaluación de las pruebas realizadas se utilizó el método de chi-cuadrado, demostrando que los operadores presentan menos dificultad con el movimiento parabólico versus el rectilíneo

Palabras-claves: Grúas RTG, gantry; hoist, simulación, operarios.

\section{INTRODUCCIÓN}

Los puertos son puntos de convergencia entre las modalidades terrestres, marítimos y aéreos, por lo cual la optimización en los puertos tiene un papel importante en la reducción de los costos de transporte (Clark, Dollar, \& Micco, 2004). En Panamá, se puede señalar que la actividad portuaria se inicia con la apertura del Ferrocarril Transístmico, el primero en el continente y se magnifica con la inauguración y posterior operación del canal de Panamá (Calvo, 1999). La revolución del uso del contenedor y la incorporación de grúas para el movimiento de estos en la década de los años cincuenta causó una auténtica revolución en el diseño de los puertos existentes (Palmer, 1999). Actualmente los puertos solicitan a los fabricantes de grúas portuarias, la incorporación de sistemas informáticos para la medición del rendimiento de estos equipos. Para tal fin se aplican diferentes indicadores de productividad y utilización como parte de este análisis (Jo \& Kim, 2019), debido a que las grúas miden el resultado del uso de ellas, pero no personalizan la medición a cada uno de los operadores de grúas que, por intervalos cortos de tiempo, utilizan estos equipos para realizar sus labores cotidianas de movilización de la carga en el patio (Esmer, 2008).

Cabe señalar que en la última década, las terminales portuarias en su afán por ser más eficientes destinan recursos para la formación del personal, aplicando simuladores de equipos (Bruzzone et al., 2011). Estos equipos permitan acelerar el proceso de capacitación de los operadores e incrementar los indicadores de productividad y Panamá no ha sido la excepción en el uso de esta tecnología (Criscione, 2018) a través Instituto Nacional de Formación Profesional y Capacitación para el Desarrollo Humano (INADEH) (Yaissel Urieta Moreno, 2018). 


\section{ciki}

X Congreso internacional de conocimiento e innovación
Ciudad del Saber, Panamá

19 y 20 de noviembre 2020

Actualmente los procesos de capacitación de los operadores de grúas incluyen la medición de los movimientos que principalmente consisten en trasladar los contenedores desde el camión al patio y viceversa (Morrish, 1996).

La Figura 1 muestra una grúa pórtico de patio sobre neumáticos de goma o en inglés rubber tyred gantry (RTG) con el detalle de sus principales movimientos tales como 1) Trolley - Movimiento horizontal X de traslado. 2) Hoist - Movimiento vertical Y de izaje de la carga. 3) Gantry - Movimiento de traslación Z de la grúa a lo largo del muelle.

Figura 1 - Partes y movimientos principales de una grúa RTG.

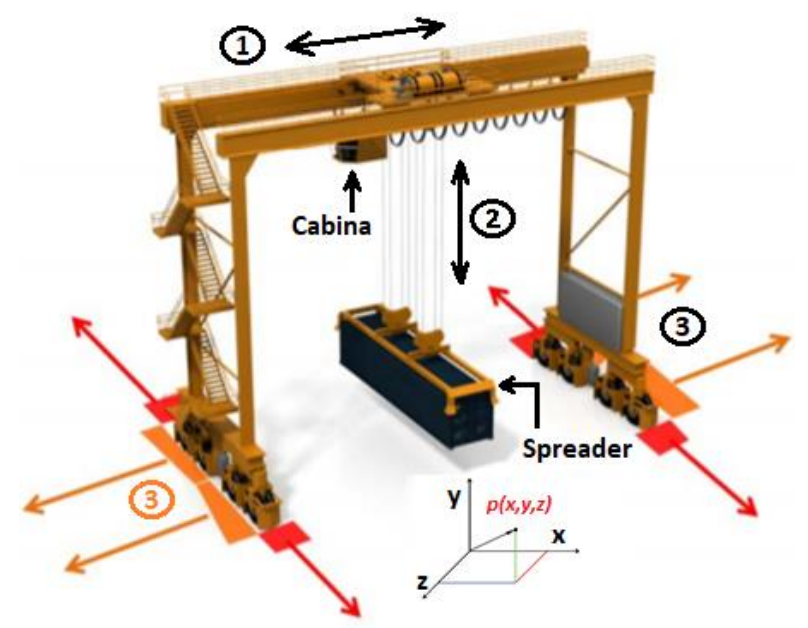

El movimiento de traslado de los contenedores a las pilas o grupo de contenedores conocido como movimiento de una mano o en forma de U invertida (rectilínea) lo que hace más lento el proceso (Papaioannou, Pietrosanti, Holderbaum, Becerra, \& Mayer, 2017).

Las nuevas corrientes de productividad en los terminales portuarios están cambiando este esquema de operación y apuntan a realizar maniobras en las que se puedan realizar ambos movimientos (trolley-hoist) de forma simultánea. Este cambio se efectúa para acortar la distancia e impactar el tiempo empleado en las maniobras, aumentando la cantidad de contenedores que se puedan mover en el tiempo determinado y por lo tanto mejora la productividad (KosucKi, MAlentA, \& ZAcZyńsKi, 2020). 
Esta investigación busca establecer las diferencias entre el movimiento de U invertida (rectilínea) y el movimiento de Overlapping (parabólica). Estos indicadores de productividad se han modelado matemáticamente y se han integrado en el proceso de capacitación y así obtener la información que nos generarán los indicadores. Se ha tomado como referencia el simulador de grúa pórtico de patio sobre neumáticos de goma - RTG (por sus siglas en inglés) que dará el reporte del ejercicio realizado por el participante utilizando información basados en el movimiento de U invertida (rectilínea) y sobre esta información establecer el modelo matemático correspondiente que emplearemos para una evaluación técnica basada en el movimiento de overlapping (parabólica), en un simulador de Grúas RTG.

\section{MATERIALES Y MÉTODOS}

Mejorar la eficiencia de los puertos de carga de contendedores ha sido un tema que el gobierno de Panamá no ha ignorado y como alternativa a este desafío crea en el año 2012 un Centro de Formación de Operadores Portuarios regido por el Instituto Nacional de Formación Profesional y Capacitación para el Desarrollo Humano (INADEH) (V., 2019)(Lozano, 2015). Este centro está en un área especial y tiene la capacidad para formar operadores en las distintas clases de equipos portuarios especializados, siendo este centro el más grande de América a nivel estatal y un fuerte contendiente para competir a nivel mundial contribuyendo a la formación de un personal capacitado en la operación de grúas portuarias en el caso que nos ocupa las grúas pórticos de patio sobre neumáticos de goma conocida como RTG (Moreno, Yaissel Urieta, 2018).

Tabla 1 - Cantidad de grúas RTG en los puertos de Panamá.

\begin{tabular}{ll}
\hline \multicolumn{1}{c}{ Nombres de los puertos en Panamá } & RTGs \\
\hline \hline PSA Panama International Terminal & 9 \\
\hline Manzanillo International Terminal & 24 \\
\hline Colon Container Terminal & 30 \\
\hline Cristóbal & 36 \\
\hline Balboa & 47 \\
\hline
\end{tabular}


El Simulador e-Tech de la grúa RTG es una herramienta que forma parte de las Tecnologías de la Información y la Comunicación (TICs) y es fundamental para el desarrollo de habilidades en la la manipulación de contenedores en una terminal portuaria. El simulador genera la dificultad propia del trabajo real con contenedores permitiendo el traslado de la carga en línea frontal sobre un camino horizontal, añadiendo las intersecciones durante el recorrido a fin que el operario preste la debida atención al tráfico de camiones.

\subsection{VARIABLES}

Las variables a estudiar son generadas por el simulador y están divididas en dos grupos. Las independientes que pueden ser modificadas y las dependientes cuyo comportamiento es influenciado por las anteriores.

Las variables independientes en este estudio son las siguientes:

1) Reportes de los simuladores

2) Cantidad de movimientos por hora

3) Productividad del operador - empresa

Las variables dependientes identificadas se listan a continuación

4) Movimiento de Trolley $=x$

5) Movimiento de Hoist $=y$

6) Movimiento de Gantry $=z$

7) Tiempo $=t$

8) Distancia recorrida $=d$

9) Velocidad $=v$

10) Oscilación $=o$

11) Altura $=h$

\subsection{CRITERIOS DE EVALUACIÓN}


Para determinar la pertinencia del análisis y la eficiencia del equipo se utilizarán parámetros que marcan rangos de desplazamiento y velocidad.

\subsubsection{Oscilación del Spreader vs Velocidad del Trolley X.}

Establece como parámetro el desplazamiento óptimo (menos de $0.75 \mathrm{~m}$ ) y máximo (3.0m) permitido de acuerdo con el diseño del equipo, basados en la oscilación del spreader al momento de desplazarse sobre el eje X (movimiento de trolley).

\subsubsection{Oscilación del Spreader vs Velocidad del Hoist Y}

Establece como parámetro el desplazamiento óptimo (menos de $1.0 \mathrm{~m}$ ) y máximo (4.0m) permitido de acuerdo con el diseño de los cables de hoist en el equipo, basados en la oscilación del spreader al momento de desplazarse sobre el eje Y (movimiento de hoist).

\subsubsection{Oscilación del Spreader vs Velocidad del Gantry Z.}

El presente criterio tiene como parámetro el desplazamiento óptimo (menos de $0.1 \mathrm{~m}$ ) permitido de acuerdo con el diseño del equipo, basados en la oscilación del spreader al momento de desplazarse sobre el eje Z (movimiento de Gantry).

\subsubsection{Resumen de movimiento de los joysticks}

El parámetro de este criterio establece el porcentaje de uso de ambas manos, basados en aplicar el movimiento de trolley y hoist de modo simultáneo (overlapping), la mayor cantidad de tiempo posible dados el porcentaje de $50 \%$ o mayor como optimo y menor de $21 \%$ como no satisfactorio, del periodo de tiempo que dure el ejercicio dado.

\subsubsection{Estadísticas de resumen del tiempo.}


El parámetro de este criterio establece que un recorrido total (ciclo) que realiza un operador, para que se considere óptimo no debe exceder los 3 minutos. Considerando que existen ejercicios donde la operación no es cómoda y exige maniobras adicionales que no se observan regularmente en la operación real.

\subsubsection{Altura del spreader.}

Indica a que altura está el spreader vs la cabina. La atura segura y razonable mínima está entre los 2 y 3 metros de altura sobre el punto más alto de la estiba de contenedores. Las fluctuaciones en las alturas son un criterio que se busca optimizar para obtener un mejor rendimiento del operador de la grúa.

\subsubsection{Trayectoria del spreader.}

Este criterio tiene como parámetro verifica la trayectoria recorrida para conocer que mantiene el mismo ritmo, dirección, altura y precisión a la hora de aproximarse a los contenedores. En ella se manifiesta el grado de efectividad de las maniobras y nos permite ver de modo general si el participante, está cumpliendo con los objetivos propios de la grúa. Cada ejercicio tiene su particularidad en cuanto a la altura óptima y el modo correcto para entenderlo es a través del conocimiento propio de las tareas a desarrollar.

Se aplica al final un criterio evaluativo sumativo cuya promoción mínima es 71. Esto implica que se debe aprobar como evaluación regular mínima en al menos 5 categorías de las siete presentadas para considerarse la aprobación mínima requerida para un estudiante de operación de grúas pórticos RTG.

\subsection{MODELO RECTILÍNEO O DE MOVIMIENTO SIMPLE DE UNA GRÚA TIPO RTG} (HOIST) 
Modelo para determinar la distancia máxima de recorrido y el tiempo mínimo desarrollado por los motores del equipo, aplicados en el simulador para las operaciones de grúas tipo RTG en forma rectilínea.

- Distancia de recorrido vertical equivale a lo siguiente: altura operacional para el movimiento de hoist en ambas direcciones, subir y bajar 21 metros hacia cada sentido.

$$
d_{1}=84 m
$$

- Distancia de recorrido horizontal equivale a lo siguiente: recorrido h operacional para el movimiento de trolley en ambas direcciones, hacia adelante y atrás 23.47 metros hacia cada sentido.

$$
d_{2}=46.94 m
$$

- Velocidad máxima del movimiento de hoist.

$$
V_{1}=45 \mathrm{~m} / \min =0.75 \mathrm{~m} / \mathrm{s}
$$

- Velocidad máxima del movimiento de trolley.

$$
V_{1}=70 \mathrm{~m} / \min =0.66 \mathrm{~m} / \mathrm{s}
$$

- Distancia total del recorrido.

$$
d=130.94 m
$$

- Velocidad total del recorrido.

$$
v=1.41 \mathrm{~m} / \mathrm{s}
$$

- Tiempo total del recorrido.

$$
t=d / v=130.94 \mathrm{~m} / 1.41 \mathrm{~m} / \mathrm{s}=92.86 \mathrm{~s}=1^{\prime} 32^{\prime}
$$

- El recorrido total rectilíneo (Ciclo), un movimiento a la vez equivale en tiempo a 1'32”. (1 min $32 \mathrm{seg})$ 
Los valores del equipo, distancia de recorrido, velocidades de cada uno de los movimientos, permite establecer una base para comprobar el comportamiento de cada operador en función del ejercicio que realiza.

El simulador basado en esta información revela los datos obtenidos de cada participante y permite que se pueda interpretar los informes, a partir de unos criterios antes descritos.

La fuente de información de los criterios, se basan en los componentes mecánicos (poleas, cables de acero, motores eléctricos, transmisiones y otros) que son parte del diseño de la grúa tipo RTG y provistos por los manuales del fabricante.

\subsection{MODELO RECTILÍNEO DE ANÁLISIS MATEMÁTICO DE LAS OPERACIONES DE GRÚAS TIPO RTG}

Se toma en cuenta una variable en cada movimiento. Ver Figura 2 para conocer el detalle de los movimientos.

- El operador utiliza un solo joystick a la vez.

- La manera de operar la grúa es más segura.

- Un mínimo de riesgo de accidente laboral.

- Productividad más baja al mover contenedores.

- Se utilizan fórmulas de movimiento rectilíneo.

$$
d=v \cdot t \quad t=\frac{d}{v} \quad v=\frac{d}{t}
$$

Figura 2 - Operación de la grúa en forma rectilínea.

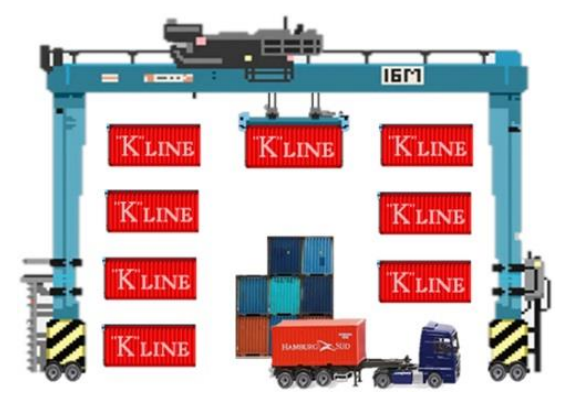




\subsection{MODELO PARABÓLICO DE ANÁLISIS MATEMÁTICO DE LAS OPERACIONES DE GRÚAS TIPO RTG (HOIST/TROLLEY)}

Se toma en cuenta en este caso dos variables en cada movimiento del operador, ver Figura 3.

- Utiliza ambos Joystick en ciertos momentos.

- Operación no segura; se aproxima al contenedor.

- Mayor riesgo de accidente laboral.

- Productividad alta al mover contenedores.

- Se utilizan fórmulas de movimiento parabólico.

Tabla 2 - Descripción de las fórmulas utilizadas para describir el movimiento

\begin{tabular}{|c|c|}
\hline \multirow{4}{*}{$\stackrel{x}{\stackrel{\infty}{\omega}}$} & $x=v_{0} \cos \theta t($ posición $)$ \\
\hline & $v_{x}=v_{0} \cos \theta($ velocidad $)$ \\
\hline & $a=0($ aceleracion $)$ \\
\hline & $x_{\max }=\frac{2 v_{0}^{2} \operatorname{sen} \theta \cos \theta}{g}=\frac{v_{0}^{2} \operatorname{sen} 2 \theta}{g}$ \\
\hline \multirow{6}{*}{ 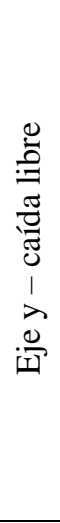 } & $v_{y}=v_{0} \operatorname{sen} \theta \pm g t($ velocidad $)$ \\
\hline & $T_{\text {vuelo }}=\frac{2 v_{0}^{2} \operatorname{sen} \theta}{g}$ \\
\hline & $a=-g($ aceleración $)$ \\
\hline & $y=v_{0} \operatorname{sen} \theta \pm \frac{1}{2} g t^{2}($ posición $)$ \\
\hline & $\tan \theta=\frac{4 y_{\max }}{x_{\max }}($ inclinación $)$ \\
\hline & $y_{\max }=\frac{v_{0}{ }^{2} \operatorname{sen}^{2} \theta}{2 g}($ Altura máxima $)$ \\
\hline
\end{tabular}




\section{ciki}

X Congreso internacional de conocimiento e innovación
Ciudad del Saber, Panamá

19 y 20 de noviembre 2020

Figura 3 - Operación de la grúa en forma parabólica.

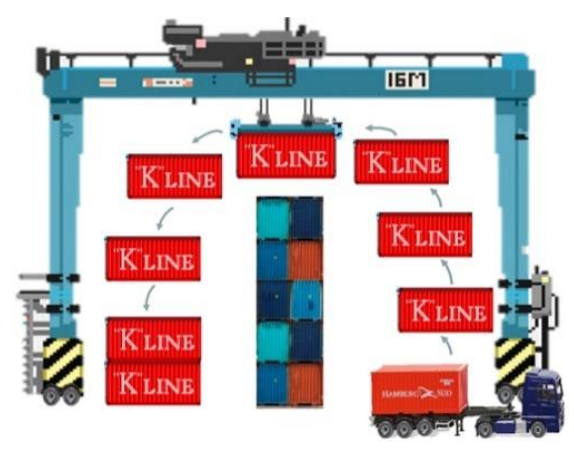

\subsection{MODELO PARABÓLICO DE ANÁLISIS MATEMÁTICO DEL SIMULADOR E- TECH SIMULATION RTG}

El Simulador e-Tech de una Grúa RTG es una herramienta clave para el desarrollo de habilidades en la manipulación de contenedores en una terminal portuaria ${ }^{5}$.

Los simuladores de maquinaria portuaria de e-Tech tienen la tecnología necesaria para que la simulación como medio de formación y aprendizaje alcance su máximo aprovechamiento. Con esta tecnología formamos operadores prácticos que tendrán la capacidad de controlar cualquier situación de riesgo debido a que se entrenaron en ambientes seguros que replican vivencias reales, accidentes, cambios climatológicos y daños de equipos para que aprendan los protocolos a seguir en cada uno de los mismos.

\section{RESULTADOS Y DISCUSIÓN}

Desde esta perspectiva cabe señalar que nuestro país cuenta con cuatro (4) grandes transnacionales que operan cinco puertos principales de manejo de contenedores con capital humano aproximado de 2,500 de los cuales el 75\% representa los operadores para los distintos tipos de equipos del sector portuario de manipulación de carga general y contenerizada. La

\footnotetext{
5 (http://www.etechsimulation.com/index.php/industrias/simuladores-portuarios/grua-rtg).
}

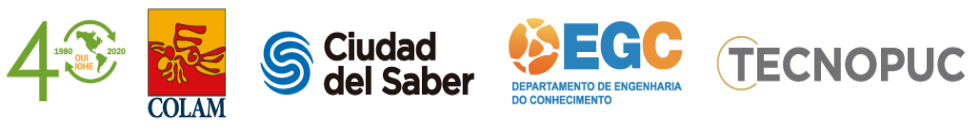


información técnica, el perfil del futuro colaborador y sus funciones específicas, los elementos e instrumentos necesarios para que se desarrolle en esta actividad, son parte del impacto del desarrollo de esta propuesta de proyecto a desarrollar.

\subsection{RESULTADOS DEL USO DEL SIMULADOR}

Teniendo en cuenta, las características de las operaciones de las grúas RTG, se realizó un estudio de caso para una simulación de uno de los puertos de Panamá, en este caso se tomó como estudio de caso un operador, sobre el uso del joysticks en las operaciones, ver figura 5.

El uso en paralelo de ambos joysticks es una garantía de que se está realizando un movimiento parabólico por lo cual es un criterio relevante para la valorar el desempeño del operador.

En el caso que solo utilice un joystick denota que solo está realizando uno de los movimientos por el cual el resultado será rectilíneo.

Figura 4 - Resultados del uso del simulador.

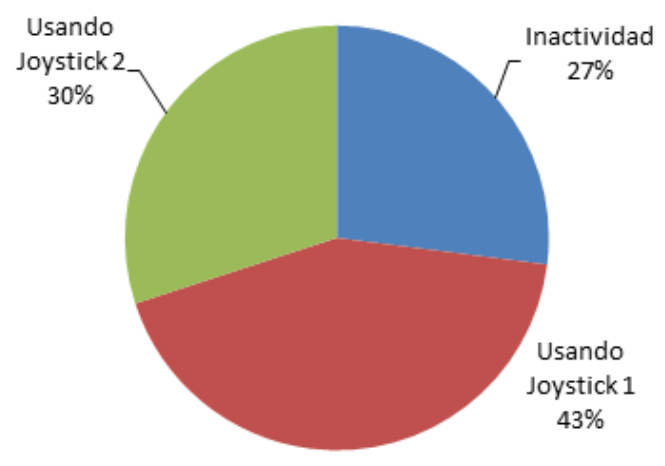

Luego se seleccionaron 120 operadores portuarios de forma aleatoria para que realizaran los ejercicios de simulación, usando movimiento rectilíneo y movimiento parabólico, para los cuales se obtuvo el siguiente resultado, en la Tabla 3: 
Tabla 3 - Resultados de las evaluaciones de los operadores.

\begin{tabular}{lllllll}
\hline Movimiento & E & MB & B & R & NS & Total \\
\hline \hline Rectilíneo & 40 & 20 & 20 & 30 & 10 & 120 \\
\hline Parabólico & 60 & 30 & 15 & 10 & 5 & 120 \\
\hline Total & $\mathbf{1 0 0}$ & $\mathbf{5 0}$ & $\mathbf{3 5}$ & $\mathbf{4 0}$ & $\mathbf{1 5}$ & $\mathbf{2 4 0}$
\end{tabular}

Como se muestra en este estudio en el simulador, los operarios cuando utilizan el movimiento parabólico, tienen mejores resultados que cuando utilizan el movimiento rectilíneo como muestra la Figura 5.

Figura 5 - Agrupación de los resultados de los movimientos.

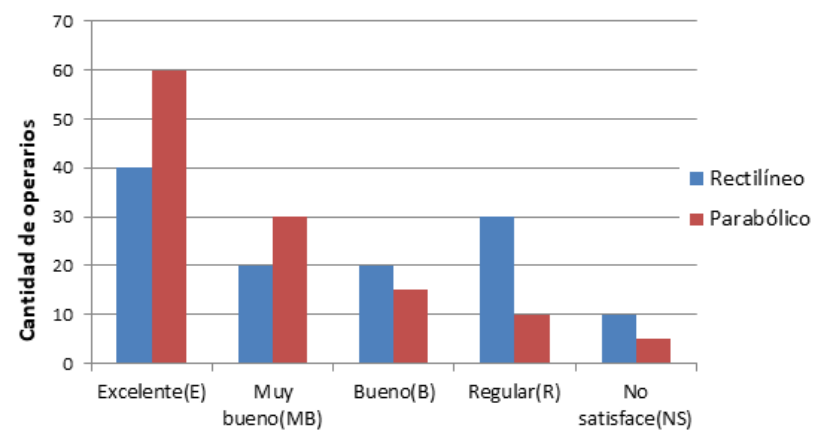

Luego se agruparon según los resultados en los que no tiene dificultad el movimiento (E, MB, B) y los que sí la tienen(R y NS), quedando dos categorías que se puede observar en la Tabla 4.

Tabla 4 - Resultados de la dificultad percibida por los operadores.

\begin{tabular}{ccc}
\hline Movimiento & No tienen dificultad & Si tienen dificultad \\
\hline \hline Rectilíneo & 80 & 40 \\
\hline Parabólico & 105 & 15 \\
\hline
\end{tabular}

Para analizar si existen diferencias significativas entre los diferentes grupos de estudiantes de los diferentes cursos se realizó las comparaciones de los mismos a través de 
pruebas de independencias de Chi-cuadrado $\left(\chi^{2}\right)$. Esta prueba contrasta frecuencias observadas con las frecuencias esperadas de acuerdo con la hipótesis nula (F.R.S., 1900), implementados en script escritos en el lenguaje estadístico R.

Para determinar la asociación o independencia de dos variables cualitativas, se realiza el test de chi-cuadrado $\left(\chi^{2}\right)$, Este test contrasta dos hipótesis, una nula $\left(\mathrm{H}_{0}\right)$ y otra alternativa $\left(\mathrm{H}_{1}\right)$.

Hipótesis nula, Ho: No hubo diferencia significativa entre la proporción los operarios que tienen dificultad y los que no usando los movimientos rectilíneo y parabólico.

Hipótesis alternativa, H1: Los operarios que usan el movimiento rectilíneo tuvo una menor proporción sin dificultad que los operarios que usan movimiento parabólico.

Sea $\alpha=0.05$ y el número total de operarios de la muestra $M$, si el $p$-value $<\alpha$, se rechaza $\mathrm{H}_{0}$ y se acepta $\mathrm{H}_{1}$, en caso contrario se acepta $\mathrm{H}_{0}$.

Usando el lenguaje $\mathrm{R}$ se realizó una comparación para saber qué operación es la más están preparados los operadores portuarios de los puertos marítimos en Panamá.

\#Este script para realizar test de Chi-cuadrado de Pearson usando tablas de contingencias

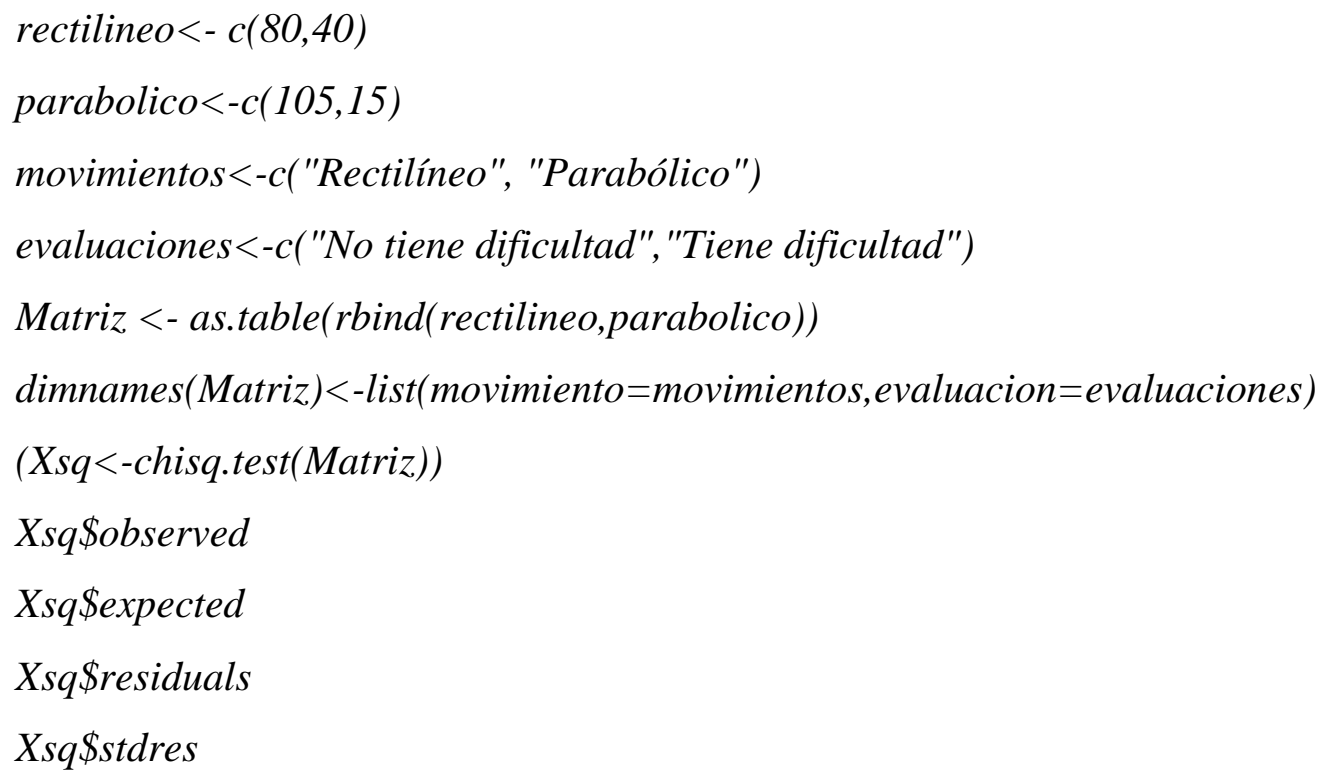

Luego se obtuvo los siguientes resultados 
Pearson's Chi-squared test with Yates' continuity correction data: Matriz X-squared $=$ 13.586, $\mathrm{df}=1, \mathrm{p}$-value $=0.0002278$.

Como se puede apreciar en la tabla anterior, el p-value es menor que $\alpha=0.05$, por lo que se rechaza $\mathrm{H}_{0}$ y se aceptó $\mathrm{H}_{1}$, concluyéndose que no tienen dificultad los operarios en usar el movimiento parabólico.

\section{CONCLUSIÓN}

Los resultados del experimento realizado con los operarios en el simulador, demostraron que los operarios presentan menos dificultad con el movimiento parabólico que con el rectilíneo. Además, se evaluó el desempeño de los operadores de las grúas tipo RTG por parte del INADEH en el área especial de la Agencia Panamá Pacífico, a través del uso del simulador de grúas RTG.

\section{AGRADECIMIENTO}

Se agradece a INADEH por proveer la información. También a la Secretaría Nacional de Ciencias y Tecnología de Panamá (SENACYT) por proveer los fondos para realizar la investigación.

\section{REFERENCIAS}

Bruzzone, A., Fadda, P., Fancello, G., Massei, M., Bocca, E., Tremori, A., ... D’Errico, G. (2011). Logistics node simulator as an enabler for supply chain development: innovative portainer simulator as the assessment tool for human factors in port cranes. Simulation, 87(10), 857-874.

Calvo, A. C. (1999). La ruta interoceánica y el Canal de Panamá. Colegio Paname no de Historiadores E Institu Panama y Estudi.

Clark, X., Dollar, D., \& Micco, A. (2004). Port efficiency, maritime transport costs, and bilateral trade. Journal of Development Economics, 75(2), 417-450. https://doi.org/https://doi.org/10.1016/j.jdeveco.2004.06.005

Criscione, J. (2018). Realidad Virtual y su aplicación como Servicios de Entrenamiento.

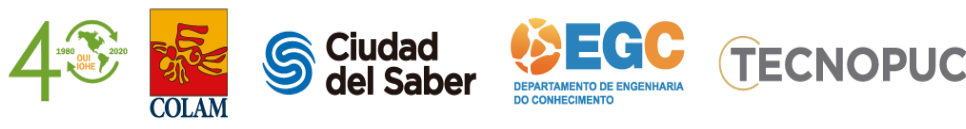




\section{ciki \\ ciki conocimiento e innovación}

Esmer, S. (2008). Performance measurements of container terminal operations.

F.R.S., K. P. (1900). X. On the criterion that a given system of deviations from the probable in the case of a correlated system of variables is such that it can be reasonably supposed to have arisen from random sampling. The London, Edinburgh, and Dublin Philosophical Magazine and Journal of Science, 50(302), 157-175. https://doi.org/10.1080/14786440009463897

Jo, \& Kim, T. (2019). Key Performance Indicator Development for Ship-to-Shore Crane Performance Assessment in Container Terminal Operations. Journal of Marine Science and Engineering, 8, 6. https://doi.org/10.3390/jmse8010006

KosucKi, A., MAlentA, P., \& ZAcZyńsKi, J. (2020). Energy Consumption and Energy Efficiency Improvement of Overhead Crane's Mechanisms. EKSPLOATACJA I NIEZAWODNOSC, 22(2), 323.

Lozano, Á. (2015, March). Panamá invierte fondos públicos en logística. Noticias Logistica y Transporte, p. 2.

Moreno, Yaissel Urieta, Y. co. (2018, July). Abre otro centro de capacitación simulada. Panamá América, p. 2.

Morrish, L. (1996). Modelling of spreader hoist systems in mobile gantry cranes.

Palmer, S. (1999). Current port trends in an historical perspective. Journal for Maritime Research, 1(1), 99-111.

Papaioannou, V., Pietrosanti, S., Holderbaum, W., Becerra, V. M., \& Mayer, R. (2017). Analysis of energy usage for RTG cranes. Energy, 125, 337-344. https://doi.org/https://doi.org/10.1016/j.energy.2017.02.122

V., D. D. (2019, November). Movimiento de carga en el Sistema Portuario Nacional registra mejora de 4.5 por ciento. Panamá América, p. 4.

Yaissel Urieta Moreno. (2018, March). Cursos del Inadeh serán obligatorios para sacar licencia. Panamá América, p. 2. 